\title{
Thanks to reviewers in 2020
}

\author{
Nicola Oberbeckmann-Winter ${ }^{1}$
}

Received: 17 September 2020 / Accepted: 18 September 2020 / Published online: 25 September 2020

(C) Springer-Verlag GmbH Germany, part of Springer Nature 2020

The mission of Analytical and Bioanalytical Chemistry (ABC) is the communication of excellent research work from all fields of (bio-)analytical chemistry. Clearly, a scientific journal's greatest responsibility toward the scientific community is to ensure that all contributions accepted for publication are rigorously but fairly reviewed in a timely manner. For this reason, all manuscripts published in $\mathrm{ABC}$ are reviewed by expert referees. The final quality of papers accepted for publication depends to a considerable extent on the reviewers, who give valuable constructive criticism to the authors and guidance to the editors. The input and dedication of all our reviewers are therefore highly appreciated by editors, authors and readers of the journal.

At the end of 2020, we gratefully acknowledge the valuable support of the following scientists who have reviewed papers for the journal during the past 12 months:

Aamouche, A.

Abad-Fuentes, A.

Abdelhamid, HN.

Ackerman, B.

Ackerman, LK.

Adam, V.

Adams, A.

Adams, FC.

Adigüzel, Y.

Afzal, A.

Aguilar-Caballos, MdlP.

Ahumada, I.

Ajayi, F.

Akgöz, M.

Akyuz, S.

Alarcón Ángeles, G.

Alben, KT.

Albert, J.

Albuquerque, CDL.

Alexandrov, T.

Alhooshani, K.

Ali, I.

Aliaño-González, MJ.
Alijanianzadeh, M.

Alonso, E.

Altmann, F.

Alvarez-Muñoz, D.

Ambrosi, E.

Amoresano, A.

Anderson, CE.

Anderson, JL.

Andersson, JT.

Anderton, C.

Anfossi, L.

Ángel Rodríguez-Delgado, M.

Annibaldi, A.

Ansell, RJ.

Antico, E.

Aramendia, M.

Arce, L.

Ariese, F.

Arigles, A.

Ariño, C.

Armenta, S.

Armirotti, A.

Armstrong, DW.
Arndt, D.

Arsene, C.

Asimakopoulos, A.

Atakay, M.

Aturki, Z.

Augusti, R.

Augusto, F.

Avino, $\mathrm{P}$.

Aw, R.

Azimzadeh, M.

Bączek, T.

Badea, M.

Baggiani, C.

Bagheri, H.

Baker, E.

Baker, LA.

Bakirhan, NK.

Bamba, T.

Banoub, J.

Bao, N.

Barber, J.

Barderas, R.

Nicola Oberbeckmann-Winter

nicola.oberbeckmann-winter@ springer.com

1 Analytical and Bioanalytical Chemistry, Springer Verlag,

Tiergartenstrasse 17, 69121 Heidelberg, Germany 
Barding, G.

Barek, J.

Bartczak, D.

Basuray, S.

Bau, HH.

Beach, DG.

Becher, F.

Becker, E.

Beitollahi, H.

Beklemishev, M.

Belder, D.

Beljebbar, A.

Benavente, F.

Benito Peña, E.

Benner Jr., BA.

Benskin, JP.

Beretta, G.

Berezin, M.

Berger, TA.

Bergquist, J.

Berlina, AN.

Bermejo Barrera, P.

Berrie, B.

Berthod, A.

Berti, F.

Bertoncello, P.

Bertrand-Michel, J.

Beverly, R.

Bezerra, G.

Bhandari, D.

Bi, X.

Biancotto, G.

Bicchi, C.

Bilewicz, R.

Birch, B.

Bishop, D.

Blanco-López, MC.

Blenner, $\mathrm{M}$.

Bocklitz, T.

Bode, $\mathrm{P}$.

Bodoki, E.

Bohn, P.

Bóka, B.

Bokhart, MT.

Bolea Fernandez, E.

Bongiorno, D.

Bonhommeau, S.

Bonner, R.

Borisov, SM.

Borsdorf, H.

Boschetti, E.

Bossi, A.

Bota, S.
Botha, A.

Bouckaert, J.

Bourgogne, E.

Bovee, TFH.

Boyaci, E.

Brajter-Toth, A.

Braun, U.

Breidbach, A.

Brezillon, S.

Brodbelt, J.

Broeckling, CD.

Brown, PN.

Brożek-Mucha, Z.

Buchberger, W.

Buckley, B.

Buckner, SW.

Bulska, E.

Bunk, DM.

Bunkoed, O.

Burmistrova, NA.

Burns, DT.

Busser, B.

Buszewski, B.

Buttinger, G.

Buttler, O.

Byrne, HJ.

Čabala, R.

Cacciola, F.

Caglayan, MG.

Cagliero, C.

Cahill, JF.

Cai, C.

Cai, Y.

Calani, L.

Calvano, CD.

Câmara, JS.

Camin, F.

Campbell, L.

Campuzano, S.

Canela-Garayoa, R.

Cantafora, AD.

Cao, $\mathrm{H}$.

Cao, X-L.

Caplan, J.

Cappa de Oliveira, LF.

Capriotti, AL.

Carasek, E.

Cárdenas Aranzana, S.

Careri, M.

Carinhanha Caldas Santos, J.

Carrasco, S.

Carrillo-Carrion, C.
Carro, AM.

Carter, C.

Caruso, F.

Caselli, F.

Castells, CB.

Cataldi, TRI.

Cauerhff, A.

Causon, T.

Cavaliere, C.

Cebolla, V.

Cela Torrijos, DC.

Cerutti, S.

Ceslova, L.

Cha, S.

Chai, Y.

Chaix, C.

Chankvetadze, B.

Chao, T-C.

Chatelut, E.

Chaurand, P.

Chemat, F.

Chen, B.

Chen, C.

Chen, D.

Chen, DDY.

Chen, FY.

Chen, G.

Chen, J.

Chen, L.

Chen, M-L.

Chen, S.

Chen, W.

Chen, $\mathrm{X}$.

Chen, Y-C.

Chen, Z.

Cheng, G.

Cheng, $\mathrm{H}$.

Cheng, Q.

Chiu, T-C.

Cho, TJ.

Choi, H-K.

Choi, JR.

Christian, R.

Christinat, N.

Christopoulos, T.

Chung, SWC.

Cialla-May, D.

Cifuentes, A.

Citterio, D.

Clark, KD.

Clowers, B.

Cobice, D.

Coelho, C. 
Coffinier, Y.

Colby, J.

Collins, B.

Cologna, SM.

Colombini, MP.

Coltro, W.

Concheiro-Guisan, M.

Conde, JP.

Conklin, DJ.

Connelly, JT.

Connolly, L.

Cooks, RG.

Cooper, WT.

Corbisier, P.

Cordero, C.

Costa-Fernández, JM.

Cottet, J.

Cox, HD.

Coy, SL.

Cozzolino, D.

Cregut, M.

Crimmins, BS.

Cristea, CV.

Cubadda, F.

Culha, M.

Cunha, S.

Cvačka, J.

da Silva, R.

Dabrio Ramos, M.

Dall'Asta, C.

Dang, F.

Danielson, N.

Das, J.

Dastan, D.

Davies, SR.

de Juan, A.

de la Rica, R.

de los Santos Álvarez, N.

De Ninno, A.

De Pauw, E.

de Santana, F.

de Villiers, A.

Dedieu, $\mathrm{M}$.

Degano, I.

Deivasigamani, $\mathrm{P}$.

Del Bubba, M.

del Olmo Iruela, M.

del Valle, M.

Delatour, V.

Delmonte, P.

Delvigne, F.
Demeke, T.

Demirev, P.

Deng, B.

Deng, C.

Deng, $\mathrm{H}$.

Deng, Q.

Deng, $\mathrm{X}$.

Deng, $\mathrm{H}$.

Depciuch, J.

Desaire, $\mathrm{H}$.

Descalzo, AB.

Desire, C.

Deventer, K.

Díaz-García, ME.

Dickert, FL.

Dierkes, G.

Dillen, L.

Dina, N.

Ding, S-N.

Ding, $Y$.

Dixon, D.

Do, TD.

Donato, P.

Dong, C.

Donnarumma, D.

D'Orazio, G.

Dornellas, RM.

Dorner, M.

Dostalek, J.

Dotsikas, Y.

Dou, M.

Drake, RR.

Du, E(S).

$\mathrm{Du}, \mathrm{J}$.

Duan, Y.

Duewer, D.

Dufva, M.

D'Ulivo, A.

Duncan, J.

Duran, $\mathrm{H}$.

Dzantiev, BB.

Dziadosz, M.

Economou, A.

Edelmann, M.

Edgar, K.

Ehrhardt, CJ.

Ekroth, S.

El Hadri, H.

El-Ahmady, S.

El-Aneed, A.

Elbadry, M.
Eletxigerra, U.

Ellis, SR.

El-Safty, S.

Emons, $\mathrm{H}$.

Emteborg, H.

Enderlein, J.

Engel, M.

Engelhard, C.

Erden, PE.

Erim, BF.

Ermini, ML.

Ertekin, K.

Esteban, M.

Esteve-Romero, J.

Esteves da Silva, JCG.

Ewing, A.

Fabbris, L.

Fakhrullin, RFU.

Fan, $\mathrm{H}$.

Fang, J.

Favretto, D.

Fechete, R.

Fechner, P.

Feldmann, J.

Feng, W.

Feng, Y-Q.

Fernandez, B.

Fernandez, JA.

Fernandez Abedul, MT.

Fernandez-Lima, F.

Ferreira, C.

Fetzer, JC.

Fiehn, O.

Fillet, M.

Fontanals, N.

Foret, F.

Fornstedt, T.

Forster, R.

Foster Mesko, MF.

Franco, R.

Fratila, RM.

Frenich, AG.

Frolov, A.

Frosch, T.

$\mathrm{Fu}, \mathrm{Z}$.

Fujii, S-I.

Fujimaki, M.

Fullwood, NJ.

Gagnon, Z.

Gallardo, E. 
Gallello, G.

Galli, R.

Gamella, M.

Gan, N.

Gao, H.

Gao, Q.

Gao, S.

Gao, X.

Gao, Z.

García-Barrera, T.

García-Campaña, AMM.

Garcia-Cordero, JL.

Gargano, A.

Garrido, M.

Garvey, J.

Gaspar, EMMSM.

Gates, M.

Gaudreau, E.

Gaulier, J-M.

Ge, G.

Gebauer, P.

Genc, R.

Geueke, B.

Ghosh, A.

Giannetti, A.

Gibson-Daw, G.

Gigante, GE.

Gilar, M.

Gilardi, G.

Giuffrida, D.

Givelet, L.

Godin, J-P.

Goenaga-Infante, $\mathrm{H}$.

Goessler, W.

Goldman, ER.

Goluch, E.

Gomara, B.

Gomez, C.

Gomez, M.

Gomez Ariza, JL.

Gong, $\mathrm{M}$.

Gonzalez-Iglesias, $\mathrm{H}$.

González-Sálamo, J.

Gonzalez-Sapienza, G.

Goodpaster, JV.

Goodwin, R.

Gorecki, T.

Gorris, HH.

Goryacheva, IY.

Goubert, G.

Gouget, AC.

Grant, DF.
Grasso, G.

Grechnikov, A.

Greener, J.

Griffin, P.

Grigoryev, A.

Gritt, F.

Gröger, TM.

Gross, JH.

Gryczynski, Z.

Gu, Y.

Guan, F.

Guijt, R.

Gundlach-Graham, A.

Guo, M.

Guo, P.

Guo, Y.

Guo, Z.

Gupta, KC.

Gupta, VK.

Gutarowska, B.

Gyurcsanyi, RE.

Haag, M.

Hackl, T.

Haddad, PR.

Haglund, P.

Hahm, G.

Hamidi, EY.

Hamm, G.

Han, D.

Han, S.

Hann, S.

Hantao, LW.

Happel, O.

Harrington, PdB.

Harris, R.

Harrison, CR.

Hasanzadeh, M.

Hassan, U.

Hauser, PC.

Hawkridge, A.

Hayen, H.

Hayes, MA.

He, L.

He, M.

He, R.

He, Y.

Heath, E.

Heberger, K.

Hecht, E.

Heeren, RMA.

Heidari, G.
Heiss, C.

Heltai, G.

Henrion, A.

Hermabessiere, L.

Hernandez, F.

Hernández Borges, J.

Hernandez-Cordoba, M.

Hernández-Mesa, M.

Herrero, M.

Herzsprung, P.

Heudi, O.

Heumann, KG.

Hildebrandt, N.

Hill, C.

Hill, S.

Hill, SJ.

Hinze, WL.

Ho, K-S.

$\mathrm{Ho}, \mathrm{SSH}$.

Hoffmann, P.

Hoijtink-Huijbrechts, AML.

Holčapek, M.

Holland, LA.

Hollender, J.

Hongsibsong, S.

Horváth, V.

Hosseini, M.

Hou, X.

Hou-Broutin, Y.

Hrobonova, K.

$\mathrm{Hu}, \mathrm{B}$.

Hu, D.

$\mathrm{Hu}, \mathrm{J}$.

$\mathrm{Hu}, \mathrm{Q}$.

$\mathrm{Hu}, \mathrm{W}$.

$\mathrm{Hu}, \mathrm{Y}$.

Huang, CZ.

Huang, F.

Huang, G.

Huang, L.

Huang, Q.

Huang, $\mathrm{X}$.

Huang, Y.

Hubert, P.

Hugelier, S.

Hughes, MP.

Hummon, AB.

Huo, F.

Hurth, C.

Hussain, G.

Hutzler, C.

Huynh, K. 
Hytönen, VP.

Igne, B.

Indrasekara, $\mathrm{S}$.

Iqbal, N.

Itoh, $\mathrm{T}$.

Ivleva, NP.

Jackson, GP.

Jackson, SN.

Jaffrezic-Renault, N.

Jahouh, F.

Jain, P.

Jakobsen, J.

Janfelt, C.

Jang, HW.

Jankowski, KJ.

Janssen, H-G.

Jelen, HH.

Jeong, J-S

Jia, L.

Jiang, C.

Jiang, $\mathrm{H}$.

Jiang, $\mathrm{T}$.

Jiang, $\mathrm{X}$.

Jiang, Y.

Jin, M.

Jin, Y.

John, G.

Johnson, M.

Johnson, RC.

Jones, JJ.

Jones, L.

Josephs, RD.

Jung, G.

Jung, J.

Jungbauer, A.

Jungnickel, G.

Jurado Sánchez, B.

Jurasekova, Z.

Kacik, F.

Kadimisetty, K.

Kafouris, D.

Kakarla, RR.

Kaljurand, M.

Kallenborn, R.

Kalogianni, DP.

Kang, J.

Kang, T.

Kapusta, P.

Kara, P.

Karabagias, IK.
Kari, N.

Karthik, K.

Kasicka, V.

Kato, M.

Kawasaki, H.

Kazarian, SG.

Ke, R.

$\mathrm{Ke}, \mathrm{S}$.

Kelly, R.

Kenessov, B.

Kerman, K.

Kersten, H.

Kessler, RW.

Kessler, W.

Khodov, I.

Khoo, K-H.

Kim, B.

Kim, M.

Kim, S.

Kirsanov, DO.

Kitagawa, S.

Klampfl, CW.

Kneipp, J.

Knepper, TP.

Knob, R.

Knochenmuss, R.

Knoll, W.

Knopp, D.

Koch, M.

Koellensperger, G.

Koerner, $\mathrm{T}$.

Köfeler, G.

Kolarich, D.

Konermann, L.

Kong, D.

Kong, KV.

Kong, X.

König, S.

Köper, I.

Kos, G.

Koschella, A.

Kosman, J.

Kostyukevich, YI.

Kramer, M.

Kranz, C.

Kratzer, J.

Krska, R.

Kruss, S.

Krylov, SN.

Kuehnelt, D.

Kuesters, M.

Kuhn, A.

Kuklenyik, Z.
Kumke, MU.

Kuo, T-R.

Kutner, W.

Laborda, F.

Lacorte, S.

Laemmerhofer, M.

Laganà, A.

Lai, W.

Lammertyn, J.

Lanças, FM.

Lanekoff, I.

Lang, BE.

Lao, W.

Laux, E-M.

Lavine, BK.

Leblanc, RM.

Lebrilla, C.

Lederer, A.

Lee, HK.

Lee, HS.

Lee, YH.

Lee, Y-J.

Legnaioli, S.

Lehmann, WD.

Lehotay, SJ.

Leitner, A.

Leng, G.

Leopold, K.

Leopold, N.

Lesot, PGJ.

Lesur, A.

Lhotská, I.

Li, B.

Li, D.

Li, D-W.

Li, F.

Li, G.

Li, H.

Li, HY.

Li, J.

Li, L.

Li, NV.

Li, W-Y.

Li, X.

Li, Y-F.

Li, Z.

Lian, H-Z.

Liang, Y.

Liebeke, M.

Lieberman, M.

Lieberzeit, PA.

Liebisch, G. 
Ligor, $\mathrm{T}$.

Lim, KF.

Limbeck, A.

Lin, C-H.

Linden, $\mathrm{R}$.

Lindholm-Sethson, BD.

Linz, T.

Lísa, M.

Lisdat, F.

Litescu, SC.

Liu, B.

Liu, F.

Liu, J.

Liu, M.

Liu, Q.

Liu, S.

Liu, X.

Liu, Y.

Liu, Z.

Llobet, E.

Lobo-Castañón, MJ.

Locatelli, M.

Loo, JA.

Lopes, MB.

Lopes, N.

Lou, X.

$\mathrm{Lu}, \mathrm{F}$.

Lu, G.

$\mathrm{Lu}, \mathrm{J}$.

Lu, N.

Lu, X.

Lu, Y.

Lubman, DM.

Lucena Rodríguez, R.

Lund, RR.

Lundberg, P.

Lurie, I.

Lv, Y.

Lynen, F.

Lyskov, N.

Ma, J.

Ma, L.

MacCuspie, RI.

Mace, CR.

Maher, W.

Maheshwari, V.

Mahmud, I.

Maiwald, M.

Malek, K.

Malenovic, A.

Malet-Martino, M.

Malhotra, BD.
Malinovskiy, DN.

Malitesta, C.

Mallard, G.

Malta Costa, L.

Man, B.

Mani, V.

Mani-Varnosfaderani, A.

Maquieira, Á.

Marce, RM.

Marchegiani, E.

Marco, M-P.

Marcus, RK.

Mariassy, M.

Marina, ML.

Marini, F.

Marken, F.

Markin, AV.

Marrazza, G.

Marshall, C.

Marshall, J.

Marshall, P.

Martin, FL.

Martín, J.

Martinez Carballo, E.

Maspoch, S.

Massolini, G.

Matraszek-Zuchowska, I.

Mavel, S.

May, JC.

Mayboroda, OA.

Mayer, M.

Mayerich, D.

Mazumder, A.

McCord, BR.

McDonnell, L.

McLean, JA.

McMillan, NJ.

Meagher, RJ.

Meermann, B.

Meija, J.

Melucci, D.

Men, D.

Meng, F.

Meng, H-M.

Meng, L.

Mercolini, L.

Mesquita, R.

Miao, P.

Michael, A.

Michalke, B.

Micheli, L.

Michelini, E.

Mihasan, M.
Mikyska, A.

Miller, AL.

Millet, M.

Minor, E.

Miranda Castro, R.

Mirsky, VM.

Mishra, NL.

Misra, BB.

Mitchell, J.

Mitić, ŽJ.

Mitulovic, G.

Mnatsakanyan, M.

Moaddel, R.

Möder, $\mathrm{M}$.

Molinié, R.

Montes-Bayón, M.

Moon, MH.

Moore, DS.

Morais, S.

Moreno Cordero, B.

Moricz, AM.

Morini, L.

Morlock, GE.

Morris, GA.

Moscone, D.

Moyano, E.

$\mathrm{Mu}, \mathrm{Y}$.

Mudalige, $\mathrm{T}$.

Mukherjee, A.

Müller, T.

Müller-Platz, C.

Muneeruddin, $\mathrm{K}$.

Murray, J.

Murray, KK.

Namjoon, C.

Nanita, SC.

Napoli, A.

Narayanasamy, S.

Näreoja, T.

Naseer, MM.

Natu, R.

Nayak, P.

Nazari, M.

Nemutlu, E.

Nerin de la Puerta, C.

Neusü $\beta$, C.

Neuzil, P.

Nevin, A.

Nguyen, DC.

Nichols, KP.

Nie, Z.

Nielen, $M$. 
Niessner, R.

Nikiforov, V.

Nitride, C.

Nobrega, JA.

Noël, M.

Nojima, Y.

Nordborg, A.

Nording, ML.

Nowak, S.

Noworyta, K.

Nugen, SR.

Numata, M.

Nuñez, O.

O’Connor, P.

Olesik, SV.

Oliveira, HM.

Oliveri, P.

Olivieri, AC.

Orellana, G.

Osawa, M.

Ouyang, J.

Ouyang, Z.

Ozalp, VC.

Ozkan, SA.

Pabst, M.

Pal, S.

Palacio Lozano, DC.

Palchetti, I.

Pallaoro, A.

Palleschi, V.

Pallua, J.

Palzer, S.

Pan, J.

Pan, S-D.

Panchagnula, V.

Panderi, IE.

Panikkanvalappil, S.

Panne, U.

Pappas, D.

Park, JP.

Park, KS.

Parker, C.

Parr, MK.

Pascale, M.

Paseiro Cerrato, R.

Patra, D.

Paxton, W.

Pedersen-Bjergaard, S.

Pedrero, M.

Pei, H.

Pellerano, RG.
Peltomaa, R.

Peña-Farfal, CG.

Peng, $\mathrm{X}$.

Percy, AJ.

Pereiro, R.

Pérez Guaita, D.

Permentier, H.

Pesek, JJ.

Peter, KT.

Petersen, E.

Pethig, R.

Petit Dominguez, MdlP.

Petrovic, M.

Phillips, MM.

Phinney, B.

Pichini, S.

Pichon, V.

Pick, H.

Picó, Y.

Pingarrón, JM.

Pino, V.

Pintea, A.

Pitchaimani, V.

Pitkänen, L.

Pittenauer, E.

Place, B.

Plenis, A.

Polesello, S.

Popov, AM.

Popp, J.

Posthuma-Trumpie, GA.

Potyrailo, RA.

Powers, R.

Prado, S.

Prati, S.

Prentice, B.

Priego-Capote, F.

Proefrock, D.

Prohaska, T.

Proll, G.

Psillakis, E.

Pujari, SP.

Purcaro, G.

Pyo, JS.

Qian, J.

Qin, L.

Qin, X.

Qin, Y.

Qiu, B.

Qiu, H.

Qiu, J.

Quintana, J.
Quintas, G.

Quirino, JP.

Rabin, I.

Radványi, D.

Raftery, DM.

Raghavan, S.

Rajabi, HR.

Ramanavicius, A.

Ramautar, R.

Ramos, L.

Ramos, RM.

Raoov, M.

Rathore, AS.

Ray, T.

Razic, S.

Reemtsma, T.

Reiner, JL.

Reischer, G.

Remcho, V.

Ren, D.

Ren, J.

Ren, K.

Ren, Y.

Renger, B.

Resano, M.

Resmini, M.

Reyns, $\mathrm{T}$.

Reyzer, ML.

Rezvani, J.

Richards Kortum, R.

Riegraf, C.

Riley, KR.

Rios-Castro, A.

Rivas, GA.

Robb, P.

Robinson, A.

Rödiger, S.

Rodushkin, I.

Rohn, S.

Romero-Gonzalez, R.

Rong, G.

Rosero, M.

Rosés, M.

Roshal, A.

Rossbach, B.

Roth, G.

Roussel, J-M.

Roy, D.

Roy-Lachapelle, A.

Rubio Bravo, S.

Ruiz Encinar, J.

Ruiz-Jiménez, J. 
Ruiz-Matute, A.

Rurack, K.

Rutledge, DN.

Ruvo, M.

Saba, A.

Sabbatini, L.

Sacre, P-Y.

Sadeghi, S.

Sadowska-Rociek, A.

Sahore, V.

Saint-Marcoux, F.

Saito, Y.

Sajid, M.

Salinas-Castillo, A.

Salvador, J-P.

Salzer, R.

Samal, SK.

Sandra, K.

Sandra, P.

Sapozhnikova, Y.

Šatínský, D.

Sayyadi, N.

Schantz, M.

Scheper, T.

Scherer, G.

Scheurer, M.

Schieber, A.

Schiel, J.

Schierbaum, K.

Schirhagl, R.

Schmid, R.

Schmidt, TC.

Schmitz, OJ.

Schneider, RJ.

Schollee, JE.

Schriemer, DC.

Schug, KA.

Schuhmann, W.

Schulte, A.

Schulze, J.

Schwudke, D.

Sciarrone, D.

Seah, MP.

Séby, F.

Seeber, R.

Segundo, MA.

Seidel, M.

Seitz, W.

Șener, E.

Sengl, M.

Sergi, M.

Sezgintürk, MK.
Shallan, A.

Shamsi, MH.

Shang, L.

Shangguan, D.

Shao, M.

Sharma, A.

Sharp, J.

Shaterian, M.

Shen, F.

Sheta, SM.

Shi, F.

Shi, H.

Shiddiky, MJA.

Shieh, D-B.

Shimma, S.

Shippy, S.

Shirkhanloo, H.

Shpigun, O.

Shrestha, NK.

Shu, Z.

Shuang, S.

Sieber, JR.

Siefe, C.

Sielemann, S.

Siesler, HW.

Sikirzhytski, V.

Siluk, D.

Simal-Gandara, J.

Simó-Alfonso, EF.

Singh, DR.

Singh, N.

Sinha Roy, K.

Sirota, TV.

Sivakumar, K.

Skopp, G.

Slobodník, J.

Smith, E.

Smoluch, M.

Snow, NH.

Snyder, NW.

Soave, M.

Sojic, N.

Sojo, LE.

Solle, D.

Soltwisch, J.

Song, $\mathrm{Y}$.

Song, Y-Y.

Soukka, T.

Soylak, M.

Spanel, P.

Spence, DM.

Spoto, G.

Stanković, DM.
Stashenko, E.

Staudacher, E.

Stefan-van Staden, R-I.

Steiner, G.

Stepien, E.

Stobiecka, M.

Stoll, D.

Stove, CP.

Stürup, S.

$\mathrm{Su}, \mathrm{L}$.

$\mathrm{Su}, \mathrm{M}$.

Su, X.

Su, Z.

Sugimoto, I.

Sugimoto, N.

Supekar, NT.

Suter, MJ-F.

Švorc, L.

Swami, N.

Sweedler, JV.

Szostak, R.

Szumski, M.

Taghdisi, SM.

Taitt, CR.

Tamer, U.

Tan, Y.

Tanabe, K.

Tang, D.

Tang, X.

Tang, Y.

Tanner, J.

Tata, A.

Tauler, R.

Tea, I.

Tengattini, S.

Teo, A.

Theodoridis, GA.

Thevis, M.

Thompson, P.

Thompson, $\mathrm{T}$.

Thurman, M.

Tian, $\mathrm{H}$.

Tirler, W.

Tobin, $\mathrm{T}$.

Toffolo, M.

Tölgyesi, Á.

Tombelli, S.

Torta, F.

Touboul, D.

Towers, MW.

Tranchida, PQ.

Traversi, R. 
Tretyakova, N.

Tripathi, KM.

Trtic-Petrovic, TM.

Trujillo-Rodríguez, MJ.

Tsargorodska, A.

Tsim, KWK.

Tsochatzis, ED.

Tsui, M.

Tsunoda, M.

Tu, A.

Turnipseed, SB.

Ugarova, NN.

Uhrin, D.

Ulber, R.

Ulmer, CZ.

Ulrich, S.

Unnikrishnan, VK.

Uslu, B.

Utczas, M.

Vadillo, JM.

Valdez, CA.

Valenti, G.

Valera, E.

van Breemen, R.

van den Berg, F.

Van Keulen, H.

Van Schepdael, A.

Vanhaecke, F.

Vanna, R.

Vardaki, M.

Vashist, SK.

Vasylieva, N.

Veeravalli, V.

Verdian, A.

Verdon, E.

Vetter, W.

Villalonga, R.

Vinković Vrček, I.

Vo-Dinh, T.

Vogel, M.

Vogeser, M.

Voitechovic, E.

Volk, DE.

Vuckovic, D.

Vyskocil, V.

Waddell Smith, R.

Wagner, M.

Walker, M.

Wallace, MAG.

Wan, D.
Wang, A-J.

Wang, B.

Wang, BM.

Wang, C.

Wang, F.

Wang, G.

Wang, $\mathrm{H}$.

Wang, H-B.

Wang, H-YJ.

Wang, J.

Wang, L.

Wang, M.

Wang, Q.

Wang, S-F.

Wang, W.

Wang, $X$.

Wang, Y.

Wang, Z.

Wark, A.

Waters, J.

Webb, ME.

Weber, G.

Weber, SG.

Wegscheider, W.

Wei, G.

Wei, H.

Wei, W.

Weidner, S.

Weimar, U.

Weller, MG.

Weller, P.

Welsch, R.

Wen, Y.

Weng, L-T.

Weng, W.

West, C.

Westerholm, R.

Wheeler, K.

Whelan, RJ.

White, I.

Wieczorek, PP.

Wietecha-Posłuszny, R.

Wilkinson, K.

Willis, P.

Wilson, WB.

Wisniewski, J.

Wolfbeis, OS.

Wongkaew, N.

Wood, L.

Workman, WE.

Wright, C.

Wrobel, K.

$\mathrm{Wu}, \mathrm{A}$.
Wu, C.

Wu, J.

Wu, P.

Wu, R.

Wu, X.

Wu, Z-Y.

Wuethrich, A.

Xianghong, W.

Xiao, $\mathrm{H}$.

Xiao, Y.

Xie, J.

Xie, Q.

Xie, $\mathrm{Y}$.

Xiong, $\mathrm{X}$.

$\mathrm{Xu}, \mathrm{D}$.

$\mathrm{Xu}, \mathrm{G}$.

$\mathrm{Xu}, \mathrm{L}$.

$\mathrm{Xu}, \mathrm{M}$.

$\mathrm{Xu}, \mathrm{T}$.

$\mathrm{Xu}, \mathrm{W}$.

$\mathrm{Xu}, \mathrm{X}$.

Xuan, X.

Xue, M.

Xue, R.

Yagati, A.

Yamada, M.

Yamashita, K.

Yañez-Sedeño Orive, $\mathrm{P}$.

Yang, B.

Yang, C.

Yang, CJ.

Yang, C-S.

Yang, F-Q.

Yang, J.

Yang, L.

Yang, M.

Yang, S.

Yang, W.

Yang, X.

Yao, B.

Yao, C.

Yao, W.

Yao, Y.

Ye, F.

Ye, Y.

Yeow, E.

Yew, JY.

Yin, P-G.

Yong, Y-C.

You, B.

You, M. 
Yu, C-J.

Yu, F-Y.

Yu, H.

Yu, LL.

Yu, Y.

Yuan, H.

Yuan, M.

Yusà, V.

Zablackis, E.

Zahavy, E.

Zahn, D.

Zaia, J.

Zanardi, C.

Zare, RN.

Zelada-Guillen, G.

Zeleny, R.

Zeng, G.

Zenkina, OV.

Zhang, B.

Zhang, $\mathrm{H}$.
Zhang, J.

Zhang, J-K.

Zhang, K.

Zhang, L.

Zhang, M.

Zhang, $\mathrm{P}$.

Zhang, Q.

Zhang, R.

Zhang, S.

Zhang, W.

Zhang, X.

Zhang, Y.

Zhang, Z.

Zhao, B.

Zhao, Q.

Zhao, S.

Zhao, W.

Zhao, Z.

Zheng, N.

Zheng, W.

Zhivkov, A.
Zhong, R-L.

Zhong, W.

Zhou, L.

Zhou, Q.

Zhou, T.

Zhou, W.

Zhou, X.

Zhu, J-J.

Zhu, W.

Zhu, X.

Zhu, Y.

Zhuo, K.

Zhuo, Y.

Zimba, P.

Zimmermann, S.

Zoccali, M.

Zulfequar, M.

Zuo, G.

Zweigenbaum, J.

Publisher's note Springer Nature remains neutral with regard to jurisdic-

tional claims in published maps and institutional affiliations. 